\title{
LÍMITES IMPLEMENTADOS POR PADRES EN LA CRIANZA DE NIÑOS DE 3 A 6 AÑOS
}

\section{Limits implemented by parents in the child-rearing of 3 to 6 years old}

\author{
María Soledad Ares* , María Adela Bertella**
}

\section{Resumen}

El presente trabajo de investigación tiene como objetivo describir las características de los límites que padres de niños de 3-6 años implementan en los procesos de crianza. Para ello, se utilizó un método empírico cualitativo y el diseño de teoría fundamentada. Se seleccionó una muestra de 10 padres y 10 madres de 25 a 35 años de edad, pertenecientes a la clase media y residentes en la Provincia de Buenos Aires, Argentina. Algunos de los hallazgos realizados coincidieron con lo planteado en previos trabajos de investigación. No obstante, se descubrieron determinadas variables y hábitos que actualmente toman relevancia, tales como nuevos objetivos que los padres consideran al momento de implementar límites, o la negociación como una constante en la interacción.

Palabras clave: Padres, crianza, niños.

\begin{abstract}
The present research work aims to describe characteristics of the Limits implemented by parents in the child-rearing of 3 to 6 years old. For that purpose, a qualitative empirical method and the design of grounded theory was used. To this end was selected a sample of 10 fathers and 10 mothers between 25 to 35 years old, belonging to the middle class and residents of Buenos Aires Province, Argentina.

Some of the findings coincided with what was stated in previous research. However certain variables and habits were discovered which nowadays have relevance, such as new objectives which parents consider at the moment of set limits or negotiation as a constant in the interaction.
\end{abstract}

Keywords: Parents, child-rearing, children.

\footnotetext{
* Psicóloga. Docente Saint Matthew’s College, Buenos Aires.msol_ares@hotmail.com

** Psicóloga. Hospital Alemán. Buenos Aires. mariaadelabertella@gmail.com
} 


\section{INTRODUCCIÓN}

La combinación entre autoridad y afecto parecería influir en el desarrollo de los niños. Muchas de las dificultades actuales en las prácticas de crianza de los hijos estarían relacionadas con la disfuncionalidad de los límites parentales (Echeverría, 2011), de modo que la escuela y otros agentes educativos habrían sido progresivamente delegados con esta responsabilidad (Bayot, Hernández Viadel \& de Julian, 2005). No obstante, se plantea que los límites tendrían una doble función, conllevando no sólo prohibición sino también afecto, e informándole al niño que ni todo está permitido, ni todo está negado; de esta forma, el límite contiene y organiza al niño (Echeverría, 2011).

El desarrollo de una persona podría explicarse mediante la relación funcional entre sus características personales, el ambiente en el cual crece y las oportunidades de desarrollo y de educación que se le brindan. Serían, pues, los padres quienes adquieren la responsabilidad de propiciar las condiciones para el crecimiento saludable de sus hijos, mediante oportunidades de desarrollo y de educación (Ramírez Tobón, 2008).

En base a lo descrito, resulta importante realizar una investigación que permita explorar cuáles son las características de los límites implementados por los padres, así como también sus creencias y representaciones en torno a los mismos. Para ello, se llevaron a cabo entrevistas semi-dirigidas a padres de niños entre 3-6 años, con la finalidad de recabar información que permitiera explorar estas características, teniendo en cuenta las variables de la comunicación, tolerancia, consistencia, respeto por la autonomía del niño y coherencia de acuerdo a su edad.

\section{MARCO TEÓRICO}

a. La importancia de la crianza en los primeros años de vida.

Gallego Betancur (2012) define a la familia como la primera fuente de socialización, dentro de la cual los niños van desarrollando su comportamiento, valores y creencias. En este primer ambiente social, los niños encuentran modelos con los cuales identificarse, lo cual resulta fundamental en su futuro, al momento de afrontar el mundo. Del mismo modo, es dentro de este contexto socializador donde los niños aprenden la forma de configuración de las relaciones sociales y los modos de comportarse en cada situación, a la vez que crean una imagen de sí mismos y del mundo, y adquieren la forma en que piensan y sienten al respecto. La familia continúa siendo el ámbito privilegiado para la crianza y la socialización de los hijos (Gallego Betancur, 2012; Musitu Ochoa \& Caba, 2001).

Asimismo, otros autores plantean que las prácticas educativas surgen en el contexto familiar y se transforman en una referencia para los niños, dando lugar a la socialización y al desarrollo de habilidades emocionales y estrategias de afrontamiento. Estas prácticas influencian el crecimiento de los niños en todos los casos, al margen de sus rasgos psicológicos personales. De esta forma, el comportamiento del niño refleja las características principales del estilo de socialización de cada familia (Darling \& Steinberg, 1993; Henao López \& García Vesga, 2011).

La socialización es definida como el proceso que los seres humanos atraviesan en el transcurso de toda su vida, al incorporarse a la sociedad y vivir en ella. No obstante, este proceso cobra especial importancia en los primeros años, ya que es en este momento cuando el niño se convierte de un integrante de la comunidad. Los procesos de socialización se promueven mediante la comunicación e interacción, y la transmisión de normas, de actitudes y de patrones de comportamiento, dando lugar a la dinámica de la vida del grupo familiar. El eje central de los procesos de socialización son las prácticas de crianza (Triana, Ávila \& Malagón, 2010).

La Real Academia Española afirma que la palabra crianza proviene del verbo criar, que significa nutrir, alimentar, instruir, educar y dirigir. Varios autores coinciden en destacar la importancia de la crianza, así como también los aspectos implicados en la misma: el cuidado, la protección, la afectividad, la socialización, la enculturación y la educación que los adultos le otorgan a sus hijos, especialmente en la primera etapa de su vida. Se resalta la importancia de estas acciones durante los primeros años por ser este período el de mayor vulnerabilidad y plasticidad en la vida de todo ser humano. De ellas depende el desarrollo saludable y la socialización del niño. Algunos de los aspectos 
más importantes de la crianza incluyen la educación y sus procedimientos: alimentación, limpieza, abrigo, salud y normas. Éstos adoptan diversas características en base al entorno cultural (Gallego Betancur, 2012; Peralta, 1996).

La familia interviene en el desarrollo socioafectivo del niño, brindando los modelos, valores, normas, roles y habilidades que se adquieren durante el período de la infancia. Este estadio se encuentra relacionado con el aprendizaje acerca del manejo y la resolución de conflictos, las habilidades sociales y adaptativas, las conductas prosociales y la regulación emocional (Cuervo Martinez, 2010). Lopes y Dixe (2012) hablan de un ejercicio parental positivo, esencial para el desarrollo saludable del niño. El ejercicio parental positivo es definido como un comportamiento por parte de los padres, que promueve la educación del niño mediante la implementación de límites, el desarrollo de una relación positiva y la estimulación de su potencial de desarrollo.

Elinterés sobrelaincidenciaque el comportamiento parental tiene sobre el desarrollo de los niños fue naturalmente tenido en cuenta por diversas escuelas, como el Conductismo y el Psicoanálisis. Mientras los conductistas se interesaron en la influencia del refuerzo ambiental sobre el desarrollo del niño, los psicoanalistas otorgaron importancia a los determinantes pulsionales, los cuales entraban en conflicto con los deseos de los padres y los requisitos sociales. Se consideraba que era la interacción entre las necesidades del niño y el ambiente, lo que determinaba las diferencias personales. Aún en la actualidad, permanecen como objeto de estudio los efectos que diferentes pautas de crianza ejercen sobre el desarrollo en la niñez (Darling \& Steinberg, 1993).

\section{a.1. Creencias, actitudes y prácticas}

Izzedin Bouquet y Pachajoa Londoño (2009) mencionan tres procesos psicosociales dentro la crianza: las pautas, las prácticas y las creencias de la crianza. Las pautas se relacionan con la normativa que los padres siguen frente al comportamiento de sus hijos, y son portadoras de implicancias y significados sociales. La cultura establece las pautas de crianza de los niños. Por su parte, las prácticas de crianza se encuentran en el contexto de las relaciones entre los integrantes de la familia, en el cual los padres cumplen un rol muy importante al educar a sus hijos. El poder que los padres ejercen sobre sus hijos y la influencia mutua, constituyen dos características fundamentales de esta relación. Las prácticas son acciones, comportamientos que los padres aprendieron a partir de su propia educación o por imitación. Por último, las creencias aluden al conocimiento sobre cómo se debe criar un niño, a las explicaciones que los padres dan sobre la forma en que moldean las acciones de sus hijos.

Solís-Cámara Reséndiz y Díaz Romero (2007) también plantean la diferencia entre creencias y prácticas de crianza. Según ellos, el término creencias es utilizado por expertos como sinónimo de actitudes, ideas y percepciones, las cuales refieren a un proceso cognitivo, es decir, a lo que piensan los padres de la formación de sus niños. Por su parte, las prácticas de crianza aluden a las acciones específicas que los padres utilizan para guiar a sus hijos hacia objetivos de socialización, tales como brindarles halagos para que alcancen nuevos aprendizajes. Así, estos autores proponen que mientras las creencias de crianza reflejan lo que los padres consideran que es importante al educar a sus hijos, las prácticas de crianza implican la aplicación de estas creencias, o el modo en que los comportamientos se llevan a cabo de acuerdo con estas creencias. De este modo, estudiar las creencias de crianza resulta muy interesante, ya que éstas representarían para los padres una guía general acerca de lo que consideran importante en la educación de sus hijos. No obstante, resulta relevante clarificar que según estos autores las creencias acerca de la crianza y las prácticas de los padres no siempre se encuentran vinculadas.

Según el modelo propuesto por Darling y Steinberg (1993), las prácticas de los padres son, en parte, el resultado de sus valores y metas orientadas a la socialización. Las prácticas parentales tienen una incidencia directa sobre el desarrollo de los comportamientos específicos del niño (tales como los resultados académicos o los modales) y sobre sus características (como la adquisición de determinados valores o un buen autoconcepto). Según lo especificado, las prácticas parentales son el mecanismo mediante el cual los padres ayudan a sus hijos a adquirir diversas metas de socialización. 
Asimismo, este modelo define el estilo parental como la constelación de actitudes que los padres comunican al niño, las cuales generan un clima emocional en el que los comportamientos parentales son expresados. Estos comportamientos incluyen tanto las conductas específicas y orientadas hacia una meta, mediante las cuales los padres llevan a cabo su función (prácticas de crianza), como también las conductas que no se encuentran orientadas hacia una meta, tales como los gestos, los cambios en el tono de voz o la expresión espontánea de alguna emoción (Darling \& Steinberg, 1993).

A partir del estudio de los comportamientos de los niños y las prácticas de crianza de sus padres, surgió un consenso acerca de la asociación entre tales variables. Se planteó que niños caracterizados como sociables, cooperadores, amigables, leales, emocionalmente estables, alegres, honestos y buenos alumnos, habían crecido en ambientes donde los padres se comportaban de un modo específico. Estos padres eran cálidos, y establecían pautas claras y racionales, a la vez que respetaban la autonomía de los niños dentro de ciertos límites, y comunicaban tanto sus expectativas como las razones detrás las mismas (Darling \& Steinberg, 1993; Symonds, 1939).

\section{a.2. Aspectos promovedores de un desarrollo} saludable

En su artículo, Fernández Beato (2009) menciona ciertos "ingredientes" que, al ser combinados de un modo equilibrado, facilitan la educación de los niños y promueven su desarrollo saludable. Los aspectos a los cuales se refiere son: afecto y comunicación, normas y límites, y autonomía. El modo de combinarlos equilibradamente depende de variables tales como: la edad del niño, su personalidad y grado de madurez, las creencias y valores de la madre y del padre. Por ello, resulta crucial que los padres muestren una cierta flexibilidad en el proceso educativo. No obstante, los aspectos mencionados son fundamentales en la relación padres-niño.

La autora mencionada previamente describe cuatro estilos educativos de los padres: democrático, autoritario, permisivo e indiferente. Resalta las ventajas de un estilo educativo democrático, el cual se basaría en la demostración de afecto, altos niveles de comunicación, normas y límites claros (justificados de un modo acorde a la edad y necesidades del niño) y control del comportamiento mediante tales normas y límites. Asimismo, plantea determinadas condiciones necesarias en la supervisión y el establecimiento de las normas y límites claros y coherentes. Éstas incluyen: motivos claros que justifiquen las normas, normas breves y fáciles de recordar, razonables y acordes a la edad del niño, explicaciones previas al establecimiento de las normas, consecuencias de fácil aplicación frente al incumplimiento de una norma, consecuencias con un valor de premio frente al cumplimiento de las mismas, incremento de la negociación a medida que crece el niño, consistencia e indiferencia ante protestas y rabietas, acuerdo entre los padres de acuerdo a las normas y aplicación de las consecuencias, entre otras (Fernández Beato, 2009).

Con respecto a la función paterna, algunos autores plantean la importancia de que se mantenga intacta en sus características fundamentales: la capacidad de pronunciar las palabras justas, los roles definidos y las sanciones que impongan la ley y marquen los límites con claridad (Politi \& Cappelletti, 2013). Baumrind (1967, 1971) muestra mediante los hallazgos de su investigación que aquellos padres y madres que aplicaban un control firme, pero acompañado de importantes demostraciones de amor, afecto y comprensión, promovían el desarrollo de niños estables, consistentes y responsables. Este tipo de características parentales correspondían a un estilo de tipo equilibrado.

Del mismo modo, otros autores afirman que aquellos padres que mantienen una relación positiva con sus hijos, que son firmes pero no demasiado controladores, y que priorizan el razonamiento y la persuasión por sobre el uso de poder, resultan más efectivos al momento de promover en sus hijos la internalización de las normas sociales (Grusec, Goodnow \& Kuczunski, 2000).

\section{b. Noción de límite.}

Los pilares sobre los cuales los padres se apoyan para llevar a cabo la socialización de sus hijos son el apoyo y el control. Mientras el apoyo se basa en la comunicación, el razonamiento, el afecto y la comprensión, el control está esencialmente relacionado 
con el mandato parental (Izzedin Bouquet \& Pachajoa Londoño, 2009).

Echeverría (2011) plantea que la noción de límite se encuentra íntimamente ligada a los procesos de continuidad de crecimiento y desarrollo, tanto en el niño como en su familia. Por ende, son dos los requisitos fundamentales para que existan procesos de continuidad: los patrones vinculares y los límites funcionales. Asimismo, Spitz (1965) resalta la importancia del concepto "no" como el tercer organizador del psiquismo. Este organizador (al igual que los dos primeros: la sonrisa social y la angustia del $8^{\circ}$ mes) emergería cuando un determinado estadio llega a su culminación, alcanzando un punto crítico. En su caso, constituiría la primer noción abstracta que el niño registra del afuera, siendo la misma capaz de inhibir una acción, de recortar una inmensidad en donde todo es potencialmente posible, para que algo pueda ser en acto.

Resulta de gran importancia mencionar que los padres utilizan determinadas estrategias de socialización para regular la conducta de sus hijos; estas estrategias dan lugar a estilos educativos parentales y su elección depende tanto de las características de los padres, como de los hijos (Ceballos \& Rodrigo, 1998). El conocimiento que los padres tienen de sus hijos los lleva a implementar diferentes formas de disciplina; del mismo modo, la eficacia del estilo educativo aplicado por ellos depende de la personalidad de los niños (Grusec, Goodnow \& Kuczunski, 2000).

Miller (1995) menciona cuatro técnicas disciplinarias de los padres: las inducciones, la afirmación de poder, la retirada de cariño, y el afecto. Las inducciones implican el uso del razonamiento por parte de los padres, quienes le explican al niño, por ejemplo, las consecuencias de su conducta inaceptable. La afirmación de poder se basa en la utilización de la fuerza, las amenazas de castigo o la retirada de privilegios, con la finalidad de fomentar la obediencia. Cuando esta técnica es utilizada de modo frecuente, parece impedir la interiorización de valores prosociales. Por su parte, la retirada de cariño corresponde a la expresión directa de enojo o desaprobación de los padres, cuando el niño se comporta de un modo indeseable. Incluye prácticas tales como ignorar o aislar al niño, además de las expresiones de disgusto, rechazo y decepción. La retirada del cariño puede facilitar una rápida conformidad con los deseos de los padres pero, al igual que la afirmación de poder, no promueve la interiorización de las normas de comportamiento. Finalmente, el afecto paterno implica un compromiso con el bienestar del niño, que refuerza las conductas deseadas y favorece una relación positiva entre padres e hijos, dando lugar a que los niños se mantengan receptivos a su influencia.

\section{b.1. Características de los límites en la actualidad}

Ciertos autores plantean las particularidades de la época actual, en la cual las relaciones padres-hijos ya no son asimétricas. Se manifiesta una tendencia al consenso, así como también actividades compartidas, mayor intimidad y la abolición del autoritarismo que caracterizaba la crianza en épocas pasadas. Se habla de padres desorientados, débiles y asustados que no logran inscribir a sus hijos en una normativa con límites, los cuales proveen seguridad y protección al niño (Politi \& Cappelletti, 2013).

Según la descripción realizada por Mosca (2008; Ravera, 2009), en la clínica de niños, los padecimientos más frecuentes que pueden observarse en esta época son los trastornos somáticos, la hiperactividad, la dispersión, las dificultades de aprendizaje, la violencia y los miedos, entre otros. Frente a esto, ella se pregunta qué es lo que está ocurriendo con la capacidad de límites construida por los padres. Ravera (2009) plantea que los padres no sabrían cómo hacer para llevar a cabo su función parental, en tanto límites y compresión de sus hijos. Mosca (2008) agrega que la diferencia simbólica entre niños y adultos involucra la capacidad de límites como una propiedad intrapsíquica esencial, siendo la misma una de las bases desde las cuales pensamos la construcción de la subjetividad en el niño. La capacidad de límites posibilita y construye un sistema de ligazones que permiten el sometimiento pulsional, generando la posibilidad inhibitoria del yo.

En la misma línea, Politi y Cappelletti (2013) mencionan determinados rasgos psicológicos o actitudes recurrentes en padres que tienen dificultades para implementar límites a sus hijos en edad escolar, logrando de este modo focalizarse en la importancia 
de la función paterna, y desculpabilizando - o quitándoles responsabilidad - a los niños en el proceso de su desarrollo. Los rasgos descubiertos incluyen: inmadurez, permisividad, indiferenciación de roles, inseguridad y miedos. Ser conscientes de tales rasgos ayuda a los padres en su función, pero no es suficiente. Los padres deben comprender y repensar sus propias actitudes, aprendiendo a conocerse mejor a ellos mismos y a sus hijos, y evaluando constantemente las habilidades sociales cruciales en su relación.

Echeverría (2011) plantea que una de las mayores dificultades actuales en el ámbito de la crianza se encuentra vinculada con la disfuncionalidad de los límites implementados por los padres. Además, resalta la importancia de estudiar sus creencias acerca del ejercicio de la autoridad y el rol que le otorgan a la comunicación.

\section{c. Objetivos de los padres en la implementación de los límites}

La acción socializadora de la familia tiene como objetivo lograr ciertas pautas de comportamiento en el niño, mediante el control y el afecto. Se busca generar un mayor nivel de socialización, responsabilidad y obediencia, así como también eludir los riesgos y fracasos (Guevara \& Barrera, 2006; Henao López \& García Vesga, 2011).

Tradicionalmente los psicólogos consideraron que el objetivo de la socialización apuntaba a una aceptación autónoma y autogenerada de los valores y normas parentales, y a la aplicación espontánea de tales valores y normas en ausencia de vigilancia o control. Fallas en la mencionada aceptación y aplicación por parte de los niños, representaban un indicador de debilidad en los procedimientos de socialización a cargo de sus padres. No obstante, en la actualidad sabemos que este objetivo de socialización no siempre es considerado, ni se lo tiene como un ideal. Los padres pueden verse satisfechos con una aceptación parcial de sus deseos por parte de los niños, con desacuerdos o modos apropiados de expresar y negociar una diferencia de posición, o con comportamientos razonables en público, aún cuando un principio se ve desestimado. Los padres perseguirían variados objetivos en base a sus percepciones de lo que es posible en diferentes situaciones, y del lugar en el que el niño se encuentra en relación al objetivo planteado (Grusec, Goodnow \& Kuczunski, 2000).
Según los autores previamente mencionados, resulta lógico que los padres distingan entre niveles de aceptación, y que tengan en mente diferentes tipos de comportamiento que se extienden desde lo que es ideal, hasta lo que es aceptable o tolerable y, finalmente, aquello que está fuera de discusión. Se plantea la importancia de que las investigaciones se orienten a analizar en qué situaciones ciertos niveles de aceptación son esperados frente a otros, y cuáles son las consecuencias de esto (Grusec, Goodnow \& Kuczunski, 2000).

\section{d. Diferencias en el establecimiento de límites de acuerdo al género}

La familia es un sistema en el que los miembros desempeñan roles de acuerdo a su condición psicológica y sociocultural. Del mismo modo, los comportamientos y patrones de crianza se encuentran íntimamente vinculados con estas condiciones. $\mathrm{Al}$ ser la familia el eje central del desarrollo de la personalidad de los niños, tanto las madres como los padres, y demás integrantes, ejercen una influencia vital y determinante en la primera infancia y en el posterior desarrollo (Triana, Ávila \& Malagón, 2010).

Los autores mencionados anteriormente llevaron a cabo una investigación sobre los patrones de crianza y cuidado en Boyacá, Colombia, y descubrieron que el rol de la mujer en los procesos de socialización y las prácticas de crianza continúa siendo una constante cultural central, en contextos urbanos y rurales. También observaron la ausencia de las responsabilidades de los padres, quienes asignaban esencialmente la crianza y el cuidado de los niños y las niñas a las mujeres (Triana, Ávila \& Malagón, 2010).

Solís-Cámara Reséndiz y Díaz Romero (2007) llevaron a cabo una investigación que buscaba establecer relaciones entre las creencias y las prácticas de crianza, y encontraron diferencias por género de los padres y sexo de los niños. Se halló una mayor consistencia entre creencias y prácticas en las madres. Del mismo modo, el acuerdo entre creencias y prácticas fue semejante en madres y padres de niñas, pero no de varones. Las principales creencias de las madres que se encontraron fueron: comunicación, apoyo, roles, límites y autonomía, y éstas se vincularon principalmente con las prácticas disciplinarias y de crianza. Por su parte, 
las principales creencias de los padres fueron: límites, roles y apoyo, y éstas se relacionaron principalmente con las prácticas disciplinarias.

\section{e. Otros antecedentes empíricos relevantes.}

Una de las investigaciones más conocidas sobre los estilos de interacción familiar son los estudios de Diana Baumrind (1967, 1971), sobre niños preescolares y sus padres. Esta investigadora define los estilos parentales como el modo manifiesto en que los padres ejercen el proceso de normatización y la forma en que manejan la autoridad frente sus hijos. A partir de esta investigación, Baumrind propuso cuatro estilos parentales: autoritario, equilibrado (o democrático), permisivo y no implicado. Se pudo apreciar que aquellos padres que utilizaban un control firme, pero acompañado de afecto y comprensión, ejercían una influencia positiva en el desarrollo de sus hijos, quienes se caracterizaban por ser niños estables, consistentes y responsables.

Asimismo, resulta útil referirnos al inventario de crianza, Parent-Child Relationship Inventory (PCRI; Gerard, 1994), adaptado por Roa y Del Barrio (2001), y descrito por Solís-Cámara Reséndiz et al. (2007) como un instrumento para evaluar la crianza por parte de padres de niños entre 3 y 15 años. Basado en enfoques racionales y empíricos, este cuestionario busca identificar dimensiones relevantes de las actitudes de los padres hacia la crianza: cómo son las relaciones padres-hijos y la calidad de las mismas. Las actitudes de crianza de los padres podrían ser descritas de acuerdo a las dimensiones de apoyo y control. Estos autores presentan el primer reactivo de cada subescala del PCRI (Apoyo, satisfacción con la crianza, participación, comunicación, establecimiento de límites, autonomía, distribución del rol y deseabilidad social), destacándose para el presente trabajo las variables de la comunicación, el establecimiento de límites, la autonomía y la distribución del rol.

Por su parte, Samaniego (2010) afirmó la existencia de una tolerancia diferencial de los padres hacia el comportamiento de sus hijos, y desarrolló una escala para evaluar esta variable: La Escala de Tolerancia Parental hacia los Comportamientos Infantiles. La escala incluye las siguientes dimensiones: intención, atribución disposicional, atribución de responsabilidad, afecto-enojo, afecto-ansiedad, expectativa de resistencia, fuerza en estilo parental, verbosidad, permisividad y reactividad excesiva. Se destaca para el presente estudio la importancia de investigar las variables afecto-enojo, afecto-ansiedad, verbosidad y permisividad.

Existen numerosos estudios que sustentan la importancia del presente trabajo (Coté, Vaillancourt, LeBlanc, Nagin \& Tremblay, 2006; Romano, Tremblay, Boulerice \& Swisher, 2005), los cuales fueron descritos por Raya, Pino y Herruzo (2009). Dichos estudios, partiendo del modelo de Darling y Steinberg (1993), han encontrado en el establecimiento de límites, o disciplina - mediante normas consistentes aplicadas por los padres e inducción razonada hacia su cumplimiento puesta en práctica por los niños - un factor protector importante ante la agresividad en la infancia.

Bayot et al. (2005) planteaban ya la importancia de llevar a cabo investigaciones y desarrollos teóricos que estudien a la familia en todos sus aspectos y dimensiones, profundizando en las actitudes y comportamientos de los padres y las madres (entre otros), y en cómo éstos influencian el comportamiento futuro y la formación de la personalidad de sus hijos. De este modo, se encontraría la forma de mejorar las actitudes de los padres y las madres, y se los podría formar y ayudar en sus tareas educativas.

\section{OBJETIVOS}

\section{a. Objetivo general}

Explorar las características de los límites que padres de niños entre 3 y 6 años implementan en la crianza y educación de sus hijos, y examinar las creencias y representaciones que los padres tienen sobre el ejercicio de la autoridad.

\section{b. Objetivos específicos}

- Diferenciar los objetivos que los padres consideran que los límites tienen en el desarrollo de sus hijos.

- Describir las situaciones en las cuales los padres creen que es importante establecer límites.

- Identificar las características de los límites que los padres implementan en la crianza y educación de 
sus hijos, en relación con las variables: comunicación, tolerancia, consistencia, respeto por la autonomía del niño y coherencia de acuerdo a su edad.

- Comparar, a partir de las prácticas de crianza, la implementación de los límites según el género de los padres.

- Explorar si existe una influencia de los rasgos personales de los niños en las características de los límites determinados por sus padres.

- Establecer si existe una relación entre las creencias y las prácticas de crianza de los padres.

\section{MÉTODO}

\section{a. Diseño}

El presente estudio se llevó a cabo utilizando un método empírico cualitativo. El diseño que guió el trabajo es el de teoría fundamentada, ya que las proposiciones teóricas surgieron de los datos obtenidos en la investigación.

\section{b. Participantes}

Para llevar a cabo el estudio, se seleccionó una muestra de 10 padres y madres (20 en total) de niños entre 3 y 6 años, de clase media, que residen en la Provincia de Buenos Aires.

\section{c. Instrumentos}

Se les realizó una entrevista semi-dirigida a cada uno de ellos (madres y padres por separado), la cual se focalizó en las variables de interés previamente planteadas: características de los límites en relación con la comunicación, tolerancia, consistencia, respeto por la autonomía del niño y coherencia de acuerdo a su edad, y creencias sobre el ejercicio de la autoridad. Para ello, se elaboró una serie de preguntas que se utilizó como guía para la evaluación de tales variables durante la entrevista. Algunas de estas preguntas estuvieron basadas en la Escala de Tolerancia Parental (Samaniego, 2010). No se utilizó la escala completa ya que la misma contiene dimensiones para un rango etario mayor. Cada entrevista tuvo una duración aproximada de 30 minutos y fue registrada por el entrevistador mediante su grabación.

\section{d. Procedimiento}

Luego de la recolección de los datos, se realizó el análisis a partir de los procedimientos delimitados por la teoría fundamentada, basados en la comparación constante y la generación de categorías.

\section{RESULTADOS}

a. Observaciones acerca de la dinámica de las entrevistas.

Con la finalidad de recabar la información necesaria, se llevaron a cabo 20 entrevistas a padres. Las entrevistas fueron realizadas de modo individual, en el domicilio de los entrevistados, y tuvieron una duración que osciló entre los 25 y los 45 minutos. Se procuró que no estuvieran presentes ambos progenitores al momento de cada toma, para poder apreciar sus diferentes puntos de vista y evitar una influencia mutua en las respuestas.

Las entrevistas llevadas a cabo fueron semidirigidas, de modo que los entrevistados delimitaron el campo de las mismas, focalizándose en aquellas variables que consideraban más relevantes o de mayor interés. No obstante, mediante la utilización de una guía de preguntas basada - parcialmente - en la Escala de Tolerancia Parental (Samaniego, 2010), se procuró obtener la mayor cantidad posible de datos requeridos. Las entrevistas fueron registradas mediante su grabación con la finalidad de recopilar la información de forma fidedigna.

\section{b. Hallazgos obtenidos.}

A continuación, se expondrán los hallazgos obtenidos a partir de las variables de estudio previamente definidas. Asimismo, se considerarán nuevas variables que surgieron a lo largo del proceso de investigación.

En primer lugar, se destaca que la mayor parte de los padres entrevistados coinciden en reconocer la importancia de la implementación de límites en la crianza de sus hijos. A modo de ejemplo, la madre de una niña de 3 años expresa: "Me parece que la puesta de límites es fundamental y, de hecho, los chicos mismos te dan las señales para que pongas el límite, de alguna manera, cuando te desafían constantemente".

La madre citada previamente, al igual que otros entrevistados, refiere también a la importancia de los dos pilares fundamentales en la crianza de los niños 
- el afecto y los límites: "Si bien los padres somos sus figuras de autoridad, también somos la figura que la protege, que la contiene, que la mima, que la cuida, que la quiere bien, digamos, que quiere lo mejor para ella."

Por otra parte, ya en el comienzo de las entrevistas la mayoría de los padres hace mención de las dificultades al momento de establecer los límites, debido a diversas razones tales como: las limitaciones en el tiempo compartido con el niño, el cansancio de los padres, los inconvenientes al intentar implementar las creencias de crianza, y los sentimientos suscitados por la puesta de límites en determinadas situaciones. En relación con las limitaciones en el tiempo compartido, la madre de una niña de 4 años refiere: "Pienso que los límites son necesarios y que son muy difíciles de llevar a cabo. Si en este caso, sos una mamá trabajadora, te cuesta mucho poner límites porque querés disfrutar a tu hijo, y por ahí le querés conceder cosas, o ciertas cuestiones o permisos, que si no se los concedés lo tenés a los gritos. Y es posible que lo tengas a los gritos esas dos horas que lo podés disfrutar porque llegaste a tu casa".

En línea con lo expresado anteriormente, se reitera en varias entrevistas la importancia que variables tales como el cansancio, o el grado de paciencia de los padres, tienen en las características de los límites establecidos. El padre de un niño de 3 años plantea: "Yo creo que, como todos, cada uno tiene su día. Los días de semana, cuando uno está más cansado, también el nene está más cansado pero uno pretende que cumpla con los límites que uno le marca. Por más que uno pueda entenderlo, también el cansancio de uno - y el día que tuvo en el trabajo, en la facultad, en donde fuese - llevan a que tenga menos paciencia con el nene. Los fines de semana suelo ser mucho más flexible". El padre de una niña de 3 años plantea que el cansancio puede influir de tal modo, que hasta puede llevar a la implementación del castigo físico: "A veces se me hace difícil, y a veces no tanto. Trato de imponer premio y castigo. Trato de no recurrir a los gritos y a la violencia, pero a veces - teniendo en cuenta lo cansado que estoy - puedo recurrir a un tirón de oreja”. Por último, en referencia al punto desarrollado, el padre de un niño de 3 años dice: "En general se ponen más límites después de las 6,7 de la tarde por cuestiones de cansancio de los padres. Yo siempre digo que soy más o menos buen padre hasta las 7. Después, hago lo que puedo".

Una excepción a lo expuesto anteriormente puede apreciarse en lo expresado por la madre de un niño de 3 años, quien prioriza la coherencia en el establecimiento de los límites por sobre su cansancio, y su grado de paciencia o tolerancia: "Trato de mantener una coherencia en el límite. Cuando yo me planteo algo, que no sea en función de mi cansancio, sino de lo que yo creo que está bien o está mal. O sea, yo puedo estar cansada y tener una paciencia determinada, y estar súper descansada y tener otra... Pero trato de que el límite sea siempre el mismo. Esté cansada o no esté cansada, trato de no condicionar el límite a eso, al límite de mi tolerancia, sino que el límite sea algo fijo".

En relación a los objetivos que los padres persiguen a través de la implementación de límites, existe un cierto disenso y gran variedad de objetivos según las creencias acerca de la crianza de los padres.

Dentro de los objetivos señalados con mayor frecuencia, pueden mencionarse los siguientes en orden de importancia: fomentar el respeto hacia los demás, educar, promover una convivencia armoniosa, alertar acerca de posibles riesgos y prevenir peligros, discernir lo que está bien de aquello que está mal, evitar dañar o lastimar a otras personas, construir relaciones positivas entre hermanos, primos y otros niños, enseñar a compartir, desalentar los berrinches en público, comportarse de modo adecuado y acorde a la edad, contribuir a los hábitos y a la rutina del hogar, valorar y cuidar los objetos propios (tales como juguetes), ser cuidadosos con la higiene personal (mediante el lavado de los dientes y al momento del baño), y colaborar con el orden en el hogar.

En este contexto, la madre de un niño de tres años expresa: "Me parece que los límites son importantes en su relación con la sociedad, digamos. Por ejemplo, él a veces contesta mal. Y bueno, a mí me parece que ahí hay que marcarle el límite (...). Además está el tema de compartir, y todo lo demás, que lo fue adquiriendo mucho en el jardín también. Pero bueno, si viene la prima a jugar a casa, obviamente que uno está tratando de marcar el límite, de que hay que compartir los 
juguetes, jugar bien, no lastimar al otro... O sea, me parece que los límites son importantes en la salud de él y en el cuidado de la salud del otro. Después me parece que, mientras no se perjudique en nada él y no perjudique a otra persona, soy un poco más flexible".

En menor proporción, ciertos padres recalcaron la importancia de ordenar a los niños, generar una figura de autoridad, compartir momentos en familia (tales como la cena), formar valores en el sentido amplio de la palabra, facilitar el diálogo, generar una confianza en el niño hacia sus padres, fomentar la autonomía al enseñar sobre los posibles riesgos y peligros, desarrollar la tolerancia a la frustración, y regular los niveles de sobreexcitación.

Con respecto a las situaciones en las cuales resulta importante establecer límites, los padres describieron las siguientes: cuando el/la niño/a corre algún riesgo o peligro, cuando le falta el respeto a otras personas o a sus propios padres, en momentos en que no quiere lavarse los dientes o bañarse, cuando lleva a cabo un berrinche o se muestra caprichoso/a, en momentos en que no quiere colaborar con el orden de los juguetes o de la casa, a la hora de hacer respetar la rutina del hogar o cuando se desobedecen reglas de convivencia, cuando el/la niño/a demanda la compra de nuevos objetos o rompe intencionalmente sus juguetes, cuando le pega a un hermano, amigo o compañero, en momentos en que no quiere compartir sus juguetes con pares, cuando se encuentra sobreexcitado/a (por ejemplo, luego de la cena en el horario de la noche), y cuando dice malas palabras. El modo en que se menciona las situaciones guarda relación con la frecuencia en que fueron relatadas por diferentes padres, es decir que están dispuestas por orden de importancia.

La madre de una niña de 4 años expresa: "Fundamentalmente lo que trato de hacer es ponerle límites cuando veo que hay una situación de peligro, más que nada, o una situación que me supere en cierto modo, o que pueda llegar a traer alguna consecuencia. Por ejemplo, cuando era chica, en momentos en que metía los dedos en el enchufe, o se tiraba de la escalera. Perdón, al principio tratábamos de ponerle límites en todo, y después nos dimos cuenta que no, que en realidad había que hacer esto que te estoy contando ahora, que era ponerle ciertos límites cuando había alguna situación de peligro, o alguna situación importante. (...) En ciertos momentos, quizás si hace berrinches, también le ponemos los límites. O, por ejemplo, cuando se encierra en la habitación y empieza a patear las puertas, o empieza a gritar, o le pega a algún compañerito del colegio, o al primo mismo; se pelean y se quieren sacar un juguete y se golpean. Ese tipo de cosas yo no las tolero porque está en juego también la seguridad del otro nene, o la de ella misma”. En la entrevista citada, al igual que en otras, se reitera la importancia de establecer límites sólo cuando resulta estrictamente necesario.

Se halló que la mayoría de los padres muestra un cierto grado de flexibilidad frente al comportamiento cotidiano de los niños en el hogar. Esta flexibilidad se ve asociada a temáticas tales como la comida, el juego y la exploración libre. En relación a la comida, algunos padres se muestran comprensivos cuando sus hijos no tienen apetito, de modo que permiten que los niños coman menos de lo esperado, o que lo hagan a la vez que juegan.

En otras situaciones, tales como el momento del baño, se hace un mayor hincapié en el cumplimiento de la norma impuesta. Algunos padres recurren directamente a la acción, metiendo a sus hijos en la bañadera aún cuando ellos se niegan a hacerlo. Tal como lo expresa uno de los padres en referencia al momento del baño: "Hay casos en los que tenés que actuar".

Con respecto a la variable de estudio de la comunicación, se halló que la mayoría de los padres explica las razones detrás de los límites establecidos, y/o las consecuencias que el incumplimiento de los límites puede acarrear. En línea con esto, la madre de una niña de 3 años expresa: "Siempre que la retamos, le explicamos cuál es el motivo por el cual no puede hacer determinada cosa. Es decir, si corre algún riesgo para ella, si es algo que ella no puede tocar porque se puede llegar a romper, o lo que fuese. (...) Le preguntamos si entendió qué es lo que estaba mal; si nos dice que sí, buenísimo, si nos dice que no, le volvemos a explicar cuál es el motivo por el cual estaba mal. Capaz ella no comprende lo que nosotros hacemos, pero creemos que el día de mañana esto le va a ayudar a procesar de algún otro modo las cosas". En relación al momento en que se lleva a cabo tal explicación, se encontraron 
ciertas diferencias, dado que algunos padres conversan con sus hijos mientras establecen el límite, y otros optan por reflexionar cuando ya ha pasado un rato.

Algunos padres coinciden en resaltar que aún luego de la conversación en que se explican los límites - y las razones detrás de los mismos - el comportamiento del niño continúa siendo similar. El padre de una niña de 4 años comenta lo siguiente: "Normalmente le hablo tranquilamente. Pero hablarle tranquilamente y explicarle las cosas, tampoco soluciona los problemas. Explicarle por qué las cosas son de determinada manera... Y, eso no ayuda a que ella colabore. Cuando ella no quiere hacer algo, no lo quiere hacer, no importa todas las razones y explicaciones que le des". De forma parecida, el padre de un niño de 4 años, afirma: "A veces antes de enojarme, yo trato de hablarle, a la par digamos. Me siento en el piso, para que me escuche y me ponga atención (...). Cuando empezamos a hablar, la charla es así: - Bueno pero no hagas así porque... Bueno, no lo hago más. Y después lo sigue haciendo. A los 10 minutos vuelve a hacerlo". Asimismo, en varias entrevistas se plantea cuán difícil es que los niños comprendan el límite mediante una conversación, sin recurrir a una acción más extrema.

No obstante, de modo generalizado, se describe un muy buen nivel de comprensión por parte de los niños frente a las explicaciones pertinentes a los límites. Al igual que la mayoría de los padres, en este caso el entrevistado describe la facilidad con la cual su hija comprende las consignas: "L es una nena súper inteligente y súper manipuladora. (...) La verdad es que en muchas de las situaciones que le voy a poner un límite, me termino riendo con ella porque no puedo creer el nivel de diálogos, o a dónde lleva las discusiones. Entonces, por ahí se me hace más fácil que el límite sea en un diálogo, porque realmente tomo consciencia de que el nivel de entendimiento que tiene es mucho más alto del que uno considera". En otro momento de la entrevista, el mismo padre afirma que sus conversaciones tienen un efecto en la niña, aunque este efecto pueda ser a largo plazo: "En muchas ocasiones no es que en la misma charla conseguís algo, pero siempre se queda regulando $\mathrm{y}$, tarde o temprano, algo de lo que charlaste decanta. Entonces, sé que lo internaliza".
En relación a la variable de la tolerancia, se pudo observar que la mayor parte de los entrevistados suele sentir un alto grado de ansiedad, enojo, y por momentos hasta rabia, cuando los niños no obedecen. Por lo tanto, y de modo generalizado, los padres suelen levantar la voz o gritar cuando sus hijos no responden del modo esperado, o cuando insisten en repetir un comportamiento que es considerado inapropiado. La tolerancia se encuentra íntimamente vinculada al momento y/o situación en que se imparte el límite, y podría depender de variables tales como el cansancio o la paciencia de los padres.

A lo largo del proceso de investigación, tomó relevancia una variable relacionada a la tolerancia, que no había sido contemplada en los objetivos del presente estudio: el castigo físico. Sin realizar una indagación al respecto, y de modo espontáneo e imprevisto, surgió en un número considerable de las entrevistas el castigo físico como una herramienta para lograr el comportamiento deseado, que parecía no poder alcanzarse mediante la comunicación con el niño y su comprensión. La madre de una niña de 4 años expresa: "Nosotros no somos unos padres pegadores, pero de tanto en tanto algún chirlo... A ver, no te queda otra. (...) Acá no es algo frecuente gracias a Dios. Pero últimamente con el tema del hermano, el otro día le pegué una palmada para que le duela lo mismo que le estaba doliendo al hermano lo que le estaba haciendo". Asimismo, la madre de otra niña de 4 años afirma: "Ahora tuve que empezar a tener que meter un zamarreo. A ver, no me gusta, no es mi idea andar pegándole ni mucho menos. Pero considero que en algunos momentos, ese tironcito de orejas... Porque sino es como que no te registran, ¿entendés? Vos le estás gritando, le estás diciendo y sigue en su capricho. Entonces en algún momento tenés que frenarlo con un tironcito de oreja, o con un zamarreo; alguna que otra vez le he metido un cachetazo, lo admito".

A su vez, se halló que el castigo físico suele aparecer acompañado de sentimientos negativos en los padres, tales la culpa, la incomodidad y la frustración. El padre de una niña de 5 años expresa: "También me pasa que la otra vez me enojé con L y le di un chirlo. Y la verdad es que era súper merecido, porque realmente se agotaron todas las instancias, realmente todas las instancias. Y L lo entendió, y la sensación fue que lo 
sintió realmente como un límite, al punto de que la veía compungida, y no era por el chirlo. Era compungida por mi enojo... Pero muy compungida. Y a la inversa, me pasó a mí de sentirme súper culposo después. Y la realidad es que después de ese momento, de verla tan compungida, me sentí mal. (...) No es la forma en que quería que se entienda cuál era el límite".

No obstante, aunque la variable del castigo físico parezca ser más frecuente de lo esperado, otros padres recalcan la importancia de evitar la violencia como una herramienta en la socialización de sus hijos. Esta postura es, por ejemplo, expresada por el padre de una niña de 3 años: "Me parece que los límites no están mal, siempre que no haya violencia física, que sea algo que se charle, se hable y que lo entienda". Adicionalmente, algunos padres le explican a sus hijos la importancia de no pegar, ni tener reacciones violentas con ellos o con sus pares.

Respecto a la variable de la consistencia, la mayor parte de los padres hace referencia a su dificultad para mantenerse consistentes luego de establecer posibles futuras consecuencias (o 'penitencias') ante comportamientos considerados inadecuados. Es decir, los padres expresan con gran frecuencia que no logran sostener una pauta o una consecuencia a lo largo del tiempo. A modo de ejemplo, el padre de un niño de 4 años afirma: "Trato de que cumpla con la pauta, pero no lo sostengo en el tiempo. Trato de que lo haga. O sea, le digo: -Sentate a comer. Lo digo cien veces: -Sentate a comer, sentate a comer. Y se sienta 5 minutos, come algo y se va a jugar. Se va yendo el rigor".

Asimismo, en muchos casos se menciona la ausencia de consecuencias frente al incumplimiento de una norma, o ante una amenaza que busca corregir el comportamiento del niño. Surgió una gran cantidad de ejemplos en relación a este punto de investigación, aunque sólo se detallan algunos de ellos. La madre de un niño de 6 años expresa: "Trato de que no, pero a veces pasa. Le digo: -Tranquilo porque no vas al cumpleaños. Y yo en mi interior sé que no lo voy a dejar sin ir al cumpleaños. A veces pasa, que decís cosas y que sabés que no las vas a cumplir". Por su parte, el padre de un niño de 4 años comenta: "Cuando le digo: -Si no comés no vamos a ver tele. Él me responde: -Bueno, como. Entonces come dos bocados, tres bocados, y bueno... Después vamos a ver la tele. Total íbamos a ir igual, es una amenaza sin sentido". Una de las madres entrevistadas utiliza la amenaza de enviar a su hija al 'cuartito de la penitencia', en relación a lo cual expresa: "Nosotros utilizamos el cuartito de la penitencia, que nunca se lleva adelante, nunca se hace. La amenaza es: -Portate bien o te vas al cuartito de la penitencia. No existe el cuartito de la penitencia... Pero, de alguna manera, es eso o pensar en el rincón”.

En la mayoría de las entrevistas realizadas, los padres comentan que se les dificulta más sostener los límites en el tiempo, y mantenerse firmes, sólidos y consistentes en su postura, que establecerlos en primer lugar. Este hallazgo se ve ejemplificado mediante lo expuesto por la madre de una niña de 4 años: "A veces es más fácil no poner límites, que ponerlos y sostenerlos. Lo que te cuesta es eso, lo que te cuesta es sostenerlo, no tanto ponerlo. Y a mí me está costando mucho últimamente, es bastante arrolladora la situación. (...) Yo, por ahí, le digo que no y al rato me supera la situación del grito, y se lo termino dando. Y sé que es pésimo eso, porque le estoy haciendo un mal a ella en realidad. Lo mejor es sostenerlo y que de eso ella saque una enseñanza, o que no lo vuelva a hacer porque ya sabe que va a tener ese límite, y que va a ser firme. No me siento firme".

Resulta relevante señalar que en la mayoría de los hogares, aunque no en todos, la mencionada firmeza en el mantenimiento de las pautas y los límites planteados, se encuentra más asociada a la figura paterna que a la materna. En relación a este tema, uno de los padres entrevistados expone lo siguiente: "Yo soy más constante, quizás, para poner los límites. Ella no tanto, pero en algún momento explota más que yo".

Son pocos los padres que plantean la utilización de consecuencias de corta duración, dada la temprana edad y limitada capacidad de compresión y elaboración de sus hijos. Estas consecuencias de menor duración parecen resultar más efectivas a los fines de poder cumplirlas y sostenerlas en el tiempo. Una de las madres entrevistadas afirma: "Siempre intentamos colocarle como consecuencias cosas que sepamos que nosotros como padres vamos a poder cumplir. Es decir, por ejemplo, no le vamos a decir: -No vas al cumpleaños de J.Porque la realidad es que sabemos que va a terminar yendo. Entonces, si le vamos a colocar 
una consecuencia, vamos a intentar que ésta pueda llegar a cumplirse. En algunas ocasiones la mandamos a su cuarto, y le decimos que se quede ahí no más de 3 o 4 minutos, porque entendemos que tampoco tiene una capacidad de procesamiento mucho mayor". De modo similar, el padre de una niña de 4 años expresa: "Si yo di una norma, intento que se cumpla. Por eso nunca pongo una penitencia tan grave, ¿entendés? Por ejemplo, por una semana no ves televisión. Sé que no la voy a poder cumplir por tiempo, por un montón de cosas. (...) Sí intento decirle algo concreto: -Bueno, hoy no mirás televisión. Y yo sé que hoy puedo controlar que no vea televisión".

Enrelaciónala variable del respetopor la autonomía del niño, si bien los padres tienen como objetivo que el niño comprenda la pauta, la cumpla y la internalice - comportándose en el futuro de un modo acorde a lo esperado - se halló un alto nivel de negociación en la implementación y el cumplimiento de los límites. La negociación estaría vinculada al grado de flexibilidad previamente mencionado frente al comportamiento cotidiano de los niños. Una de las madres ejemplifica el modo en que entabla acuerdos o "tratos" con su hijo de 4 años de la siguiente forma: "Vamos haciendo tratos continuamente, y hasta él mismo me dice: Mamá, hagamos un trato..." Asimismo, se plantea la importancia de realizar acuerdos solamente en ciertas situaciones y frente a determinados comportamientos, de modo que algunos de los límites impartidos serían considerados innegociables por los padres. En este contexto, la madre de un niño de 3 años expresa: "Hay cosas que se negocian y otras cosas que no. Por ejemplo, si yo voy al quiosco y él quiere comprar un alfajor, y yo quería que comprara un paquete de galletitas porque me parece más sano, bueno... Está bien, es como que ahí puedo llegar a negociar un poco. Ahora bien, antes de irse a dormir, se tiene que lavar los dientes. Y me puede tirar la casa abajo, pero no se va a ir a dormir sin lavarse los dientes. Y eso él lo sabe".

Respecto a la coherencia en las características de los límites establecidos de acuerdo a la edad del niño/ a, se halló que en todos los casos los padres tienen en cuenta las capacidades motoras, intelectuales y sociales de su hijo/a, al momento de fijar expectativas razonables y acordes en la implementación de los límites. Por ejemplo, la madre de una niña de 3 años expresa: "Los límites fueron aumentando a medida que fue pasando el tiempo, la realidad es ésa. Al principio, cuando ella era bebita, le imponíamos pequeños límites. Pero la realidad es que aumentaron cuando empezó a gatear, y después cuando empezó a caminar. Cuando empezó a caminar fue cuando ella podía llegar a correr un peligro por algo". Asimismo, en la mayoría de las entrevistas realizadas pudo distinguirse un incremento en los niveles de comunicación conforme a los estadios de desarrollo y maduración del niño/a.

Adicionalmente, pudo apreciarse la necesidad de modificar las estrategias utilizadas para establecer límites a medida que los niños fueron creciendo. Se halló un constante dinamismo en la puesta de límites, ya que los padres mantienen que día a día buscan nuevos modos y nuevas herramientas que les permitan alcanzar sus objetivos. Uno de los entrevistados afirma: "Me parece que los chicos van creciendo y van experimentando nuevas formas, y nosotros tenemos que ir encontrando nuevas maneras de demostrar límites".

Con respecto a la implementación de los límites según el género de los padres, en primer lugar debe destacarse que - en la mayoría de los hogares - la responsabilidad de la socialización de los niños y de la implementación de los límites recae en ambos progenitores. Es decir que de modo generalizado, tanto el padre como la madre se hacen cargo de aplicar pautas y límites durante los momentos que comparten con sus hijos. Por lo tanto, la mayor parte de los padres intenta establecer los límites en conjunto y, aunque en ocasiones existen diferencias en los puntos de vista y criterios para la puesta de límites, evitan desautorizarse mutuamente delante de los niños. Tal como lo expresa uno de los entrevistados: "Tratamos de que el mensaje sea siempre uno solo". De modo similar, la madre de un niño de 4 años mantiene: "Tratamos de poner los límites en conjunto, tratamos de que sea algo compartido. Y, si no es compartido, al menos tratamos de no desautorizarnos entre nosotros". En algunas oportunidades, se mencionó que las diferencias de criterio en relación a los límites constituyen un motivo de conflicto entre los padres, ante la imposibilidad de ponerse de acuerdo y actuar de modo consistente.

Por otro lado y de modo generalizado, se destaca la importancia que los rasgos de la personalidad del niño/ 
a tienen en las características de los límites establecidos por sus padres. En otras palabras, la personalidad de los niños parece influenciar de modo significativo el modo en que sus progenitores implementan los límites en la crianza. Se hallaron variadas descripciones respecto a este tema, destacándose la siguiente: "Intento distraer mi hija a veces, otras veces es difícil, porque no se distrae fácilmente. Tiene una personalidad muy marcada y es bastante complicado. También sé que a lo mejor hay chicos que con decirles 'basta' o 'no' alcanza. Sin embargo, ella tiene una actitud muy fuerte". Otra de la madres entrevistadas plantea una situación completamente diferente con sus dos hijos: "No necesitamos ponerle muchos límites, porque son bastante dóciles los dos, por ahora".

Finalmente, en lo que concierne a las creencias y prácticas de crianza, en muchas de las entrevistas realizadas - en especial por las madres - se menciona la gran dificultad que implica poder llevar los conocimientos acerca de los límites a la práctica. Al igual que en muchos casos, una madre afirma: "A veces uno cree que los límites son tales, está muy claro lo que uno piensa, pero cuando los vas a llevar a la práctica te das cuenta de que a lo mejor podés ceder en algunas cosas, o que ella también tiene que ceder en otras". En variadas ocasiones, se plantea la improvisación como el recurso disponible para realizar esta tarea: "Los límites no los tenemos ni pensados, los vamos manejando día a día porque van cambiando las formas de los nenes". Asimismo, se reitera lo complicado que resulta poder llevar a cabo lo deseado o esperado: "Los chicos te pasan como una aplanadora. Y el manual, este famoso manual que alguna vez por ahí tus padres te mencionaron, no se lo dan a nadie. Y uno va formándose como puede. Y es difícil..."

La noción de autoridad como imposición se ve desestimada, para promover un tipo de autoridad basado en el acompañamiento, la guía, el respeto y la educación. Gran parte de los padres entrevistados afirma que no tiene como objetivo que sus hijos cumplan las pautas u obedezcan los límites basándose exclusivamente en la idea de autoridad, o por representar ellos una figura de poder. A modo de ejemplo, la madre de un niño de 3 años plantea lo siguiente: "No me gusta ponerme en un pedestal de decir: -Bueno, vos vas a hacer lo que yo te digo porque yo soy tu madre. Me parece que mi papel como madre es educarlo a él en lo que le hace bien. Hacerle bien a él, al prójimo..." En el mismo contexto, el padre de una niña de 4 años mantiene: "Intento ser, en la medida de lo posible, democrático. No autoridad como imposición".

Al mismo tiempo, muchos de los entrevistados destacan cómo las propias experiencias vividas con los padres influencian el modo en que se comportan con sus hijos e imparten límites en sus hogares en el presente. De modo generalizado, se evita repetir comportamientos y hábitos paternos que se consideraron indeseables en el pasado, a la vez que se busca imitar aquellos considerados beneficiosos o positivos. Existe una considerable cantidad de ejemplos al respecto. Uno de los padres entrevistados afirma: "Es muy difícil ser padre y poner límites. (...) El único modelo que tengo son mis viejos. Lo que para mí no estaba bien en ese momento, intento manejarlo o controlarlo yo, y hacerlo mejor. Mi mamá me ponía muchos límites en su momento, cosa que capaz ahora viéndola un poco en perspectiva, no era necesario. Y yo me quiero despegar un poco de mi mamá con eso". En la misma línea, la madre de una niña de 3 años plantea una situación diferente: "Particularmente creo que yo soy bastante estricta porque a mí, cuando yo era chica, no me colocaron los límites que yo considero hoy, siendo madre, que me tendrían que haber colocado".

Finalmente, una de las madres entrevistadas describe detalladamente cómo la investigación acerca de los diferentes aspectos involucrados en la crianza la ayudó en el desempeño de su rol como madre: "Mi papá me compró un libro para que leyera sobre la crianza y demás. Y yo misma me puse a investigar y, bueno, cada etapa de $\mathrm{E}$ fue diferente. Me gusta mucho la teoría del apego. E fue cambiando mucho en muy poco tiempo. Desde que tiene 1 año hasta los 3 , fue cambiando mucho en su personalidad. Entonces una cosa que antes me funcionaba, después ya no me funcionó más. Y tuve que reinventarme. (...) Capaz que uno cuando es más grande, tiene otro tipo de conocimiento y se cree que sabe más. Yo la verdad que dije: No sé nada así que voy a recurrir a las fuentes".

A modo de síntesis, a continuación se recapitulan las categorías a las cuales se arribó en el presente estudio, mediante las siguientes tablas: 
Tabla 1.

\section{Variables relacionadas con las dificultades al momento de establecer los límites}

Limitaciones en el tiempo compartido con el niño.

Cansancio de los padres.

Inconvenientes al intentar implementar las creencias de crianza.

Sentimientos suscitados por la puesta de límites en determinadas situaciones.

Tabla 2.

Principales objetivos de los padres en la implementación de límites

Fomentar el respeto hacia los demás.

Educar.

Promover una convivencia armoniosa.

Alertar acerca de posibles riesgos y prevenir peligros.

Enseñar a discernir lo que está bien de lo que está mal.

Evitar dañar o lastimar a otras personas.

Construir relaciones positivas entre hermanos, primos y otros niños.

Enseñar a compartir.

Desalentar los berrinches en público.

Fomentar un comportamiento adecuado y acorde a la edad.

Contribuir a los hábitos y a la rutina del hogar.

Enseñar a valorar y cuidar los objetos propios.

Ser cuidadosos con la higiene personal.

Colaborar con el orden en el hogar.
Situaciones en las cuales los padres consideran que es importante establecer límites

El niño corre algún riesgo o peligro.

Se produce una falta de respeto hacia otras personas o hacia los propios padres.

Hay resistencia a lavarse los dientes o bañarse.

El niño realiza un berrinche en público o se mantiene en una postura caprichosa.

Hay resistencia a colaborar con el orden de los juguetes o de la casa.

Se desobedecen reglas de convivencia o no se respeta la rutina del hogar.

El niño demanda la compra de nuevos objetos o rompe intencionalmente sus juguetes.

Se lleva a cabo un comportamiento violento hacia un hermano, amigo o compañero.

El niño no quiere compartir sus juguetes con pares.

Existe un alto nivel de excitación.

Se pronuncian malas palabras. 


\section{DISCUSIÓN}

Este trabajo de investigación se propuso, en primer lugar, diferenciar los objetivos que los padres consideran que los límites tienen en la crianza de sus hijos. Conforme a lo planteado en el marco teórico, se halló que los padres orientan su tarea apoyándose en los pilares fundamentales del afecto y el control. Algunos de los objetivos descritos con mayor frecuencia coinciden con los mencionados por los autores (Guevara \& Barrera, 2006; Henao López \& García Vesga, 2011), en especial en lo referente a evitar riesgos o peligros y lograr mayores niveles de socialización.

Si bien el objetivo de la obediencia - mencionado en el apartado teórico - fue tenido en cuenta por los padres, también surgió la variable de la negociación. Es decir, de modo análogo a lo expresado por ciertos autores (Grusec, Goodnow \& Kuczunski, 2000), los padres se encontrarían satisfechos con la posibilidad de establecer acuerdos o "tratos" en base a los intereses, los gustos, las posibilidades y las capacidades de sus hijos, aunque las pautas impuestas se cumplan sólo parcialmente. En otras palabras, existe una cierta flexibilidad en la implementación y el cumplimiento de los límites, contemplando el respeto por la autonomía de los niños. Tal como se plantea en el marco teórico (Fernández Beato, 2009), esta flexibilidad resulta fundamental para promover un desarrollo saludable a lo largo del proceso educativo.

Adicionalmente, se mencionan diferentes niveles de aceptación teniendo en cuenta las situaciones y los comportamientos particulares, de modo que algunos de los límites impartidos son considerados innegociables por los padres.Este hallazgo se encontraríaíntimamente vinculado con lo planteado en el apartado teórico, donde se señalan diferentes tipos de comportamiento que se extienden desde lo que es ideal, hasta lo que es aceptable o tolerable y, por último, aquello que está fuera de discusión (Grusec, Goodnow \& Kuczunski, 2000).

De un modo acorde al estilo educativo democrático (Fernández Beato, 2009), se halló que los padres mantienen altos niveles de comunicación con sus hijos. Asimismo, tanto los niveles de comunicación, como las características de los límites, se encontrarían justificados de modo acorde al nivel de desarrollo evolutivo de los niños. Es decir, puede observarse coherencia en las características de los límites establecidos de acuerdo a la edad del niño/a, de modo que las pautas irían modificándose cualitativa y cuantitativamente con el paso del tiempo.

En lo concerniente a las consecuencias ante comportamientos considerados inapropiados, son pocos los progenitores que establecen consecuencias de fácil aplicación, como sería conveniente dentro de un estilo educativo democrático. Aún más, se descubrió en muchas de las ocasiones descriptas por los padres, la ausencia de consecuencias frente al incumplimiento de una norma. Si bien puede decirse que éste es un hallazgo generalizado, en la mayoría de los hogares serían las madres quienes encuentran mayores impedimentos para sostener las pautas y los límites planteados con firmeza. Según los autores citados, la aplicación de un control firme, pero acompañado de importantes demostraciones de amor, afecto y comprensión, promovería el desarrollo de niños estables, consistentes y responsables (Baumrind, 1967, 1971; Darling \& Steinberg, 1993; Symonds, 1939).

En relación a la variable de la tolerancia, se plantea cuán difícil les resulta a los padres que los niños obedezcan el límite mediante una conversación y sin recurrir a una acción más extrema, como gritar o pegar. Se halló que la mayor parte de los entrevistados suele sentir un alto grado de ansiedad y/o enojo cuando los niños no obedecen, y sería muy frecuente que los padres levanten la voz, griten o hasta utilicen el castigo físico cuando sus hijos no responden del modo esperado. De acuerdo a lo expresado en el marco teórico (Miller, 1995), si bien esta estrategia de afirmación de poder puede resultar en una rápida conformidad con los deseos o pautas, la misma impediría la interiorización de las normas sociales, a la vez que empobrecería la relación con los padres. No obstante, de acuerdo a la clasificación de las técnicas disciplinarias desarrollada por Miller, puede decirse que las estrategias de socialización mayormente utilizadas por los entrevistados corresponden a las inducciones y al afecto paterno, las cuales promueven la internalización de valores prosociales. 
Asimismo, se descubrió que las estrategias de socialización utilizadas por los padres se verían influenciadas por las características de la personalidad de sus hijos y sus comportamientos específicos, hallazgo que coincide con diversos autores (Ceballos \& Rodrigo, 1998; Grusec, Goodnow \& Kuczunski, 2000).

Respecto a la implementación de los límites según el género de los padres, se halló que la mayoría de las parejas intenta establecer las pautas y normas de educación en conjunto. Este descubrimiento difiere con lo establecido por ciertos autores, quienes plantean que la mujer aún mantiene el rol cultural central en los procesos de socialización, y que los padres asignan esencialmente la crianza y el cuidado de los niños a las mujeres (Triana, Ávila \& Malagón, 2010).

De acuerdo a las particularidades de nuestra época, vinculadas especialmente a la abolición del autoritarismo (Politi \& Cappelletti, 2013), los entrevistados refieren que se inclinan hacia un concepto de autoridad basado en el acompañamiento, la guía y el respeto, oponiéndose a la noción de autoridad por imposición.

Además, en relación a las condiciones específicas que podrían influenciar la elección de métodos, se halló la importancia que el cansancio y el grado de paciencia de los padres tienen en las características de los límites establecidos. De tal modo, la rigurosidad en la implementación de las normas se incrementaría considerablemente cuando los padres se encuentran agotados, por ejemplo, luego de una extensa jornada laboral.

En referencia a las creencias y prácticas de crianza, se encontraron impedimentos por parte de los padres para llevar los conocimientos acerca de los límites a la práctica. De modo análogo a lo planteado (SolísCámara Reséndiz \& Díaz Romero, 2007), resultó interesante estudiar las creencias acerca de la crianza, ya que éstas representan una guía general sobre lo que se considera importante en la educación y socialización de los hijos. No obstante, las creencias y las prácticas de crianza no siempre se encuentran vinculadas, debido a las dificultades que los padres refieren para llevar la teoría a la práctica. De forma similar a lo planteado en el apartado teórico (Izzedin Bouquet \& Pachajoa Londoño, 2009), en varias ocasiones las prácticas realizadas habrían sido aprendidas a partir de la propia educación o por imitación.

\section{CONCLUSIONES}

El presente estudio tuvo como objetivo la descripción de las características de los límites que padres de niños de 3-6 años establecen en la crianza.

La mayor parte de los padres entrevistados coinciden en reconocer la importancia de los límites en la crianza de sus hijos, aunque su implementación no resulta una tarea sencilla. Las dificultades referidas responden no sólo a las particularidades de la época actual, sino también a las limitaciones en el tiempo compartido con los niños, el grado de cansancio de los padres, los impedimentos al intentar plasmar las creencias de crianza y los sentimientos suscitados por la puesta de límites en ciertas situaciones.

Respecto a los objetivos que los padres consideran importantes al momento de establecer límites, tomaron relevancia nuevos propósitos tales como enseñar acerca del valor de los objetos y el esfuerzo que implica conseguirlos, desalentar los berrinches en público y los caprichos, y enseñar a ser cuidadosos con la higiene personal. Aunque la obediencia continúa siendo un objetivo tenido en cuenta, en la actualidad surge la variable de la negociación como una constante en la interacción con los niños.

Los límites que se sostienen con más firmeza estarían vinculados a la seguridad, el cuidado del cuerpo y la higiene personal. En cambio, puede observarse una flexibilidad considerable en relación a temáticas tales como la comida, el juego y la exploración libre.

Respecto a la variable de la comunicación, se halló que los padres entablan frecuentes conversaciones con sus hijos, explicando las razones detrás de los límites y/o las consecuencias que su incumplimiento puede ocasionar. No obstante, las explicaciones no siempre se llevarían a cabo antes del establecimiento de las normas, como sería esperable. 
Uno de los hallazgos más significativos de la investigación se encuentra vinculado a la consistencia en el sostenimiento de los límites. Los padres describen cierta dificultad para mantener una pauta o una consecuencia a lo largo del tiempo y para mostrarse firmes, sólidos y consistentes en su postura. Además, la mayor parte de los entrevistados suele sentir un alto grado de ansiedad y/o enojo cuando los niños no obedecen, y se halló que el castigo físico es actualmente utilizado por muchos padres como una herramienta para lograr el comportamiento deseado. Dicha variable aparecería acompañada de sentimientos negativos en los padres, tales como la culpa, la incomodidad y la frustración.

Respecto a la implementación de los límites según el género de los padres, al presente la mayoría de las parejas intenta establecer las pautas y normas de educación en conjunto, y evita desautorizarse mutuamente delante de los niños.

Por otra parte, se halló que los padres implementan diferentes formas de disciplina en base a los rasgos de la personalidad de los niños y sus comportamientos específicos. A modo de ejemplo, aquellos entrevistados que describieron a sus hijos como niños tranquilos y dóciles, señalaron que no era necesario implementar gran cantidad de límites, ni mostrarse rigurosos para alcanzar su cumplimiento.

Finalmente, los padres refirieron que evitan repetir comportamientos $\mathrm{y}$ hábitos paternos que se consideraron indeseables en el pasado. En otras palabras, se prioriza la reflexión y la búsqueda de nuevas estrategias y modos propios de educación de los hijos.

Para concluir, los hallazgos muestran que resulta de gran relevancia continuar realizando investigaciones que brinden nuevas posibilidades y acompañamiento a los padres en su difícil tarea de educar, en especial para que eviten recurrir a estrategias indeseadas como el castigo físico. Asimismo, sería interesante continuar explorando las características de los límites en muestras de mayor amplitud, y representativas de diferentes poblaciones.

\section{REFERENCIAS}

Baumrind, D. (1967). Child care practices anteceding three patterns of preschool behavior. Genetic Psychology Monographs, 75(1), 43-88.

Baumrind, D. (1971). Current patterns of parental authority. Developmental Psychology, 4(1), 1103.

Bayot, A., Hernández Viadel, J. V. \& de Julian, L. F. (2005). Análisis factorial exploratorio y propiedades psicométricas de la escala de competencia parental percibida. Versión para padres/madres (ECPP-p). Revista Electrónica de Investigación y Evaluación Educativa, 11(2), 113126.

Ceballos, E \& Rodrigo, M. (1998). Las metas y estrategias de socialización entre padres e hijos. Madrid, España: Alianza.

Cuervo Martínez, A. (2010). Pautas de crianza y desarrollo socioafectivo en la infancia. Diversitas: Perspectivas en Psicología, 6(1), 111-121.

Darling, N. \& Steinberg, L. (1993). Parenting style as context: An integrative model. Psychological Bulletin, 113, 487-496.

Echeverría, M. L. (2011). Vicisitudes del desarrollo infantil, límites parentales y conceptualización del caso. Revista de la Asociación de Psicoterapia de la República Argentina, 2, 1-14.

Fernández Beato, M. P. (2009). Los estilos educativos de los padres y madres. Revista Digital Innovación y Experiencias Educativas, 16, 1-9.

Gallego Betancur, T. M. (2012). Familias, infancias y criaza: tejiendo humanidad. Revista Virtual Universidad Católica del Norte, 35, 63-82.

Grusec, J. E., Goodnow, J. J. \& Kuczunski, L. (2000). New Directions in Analyses of Parenting Contributions to Children's Acquisition of Values. Child Development, 71(1), 205-211.

Henao López, G. C. \& García Vesga, M. C. (2011). Interacción familiar y desarrollo emocional en niños y niñas. Revista Latinoamericana de Ciencias Sociales, niñez y juventud, 7(2), 785802.

Izzedin Bouquet, R. \& Pachajoa Londoño, A. (2009). Pautas, prácticas y creencias acerca de crianza... Ayer y hoy. Revista Liberabit 15 (2), 109-115. 
Lopes, M. S. O. C. \& Dixe, M. A. (2012). Ejercicio parental positivo por los padres de niños hasta 3 años: construcción y validación de escalas de medición. Revista Latino-Americana Enfermagen, 20(4), 1-9.

Miller, P. A. (1995). El desarrollo y la socialización de la conducta prosocial. Madrid, España: Editorial Visor.

Mosca, A. (2008). Imagen y subjetividad. Boletín Sociedad de Psicología del Uruguay, 46, 8-13.

Musitu Ochoa, G. \& Caba, M. J. (2001). La familia y la educación. Barcelona, España: Octaedro.

Peralta Espinoza, M. V. (1996). La crianza de los niños menores de seis años en Latinoamérica. Santiago de Chile, Chile: Organización de Estados Americanos.

Politi, M. E. \& Cappelletti, A. (2013). Rasgos psicológicos comunes en padres con dificultades en establecer límites. Tesis no publicada, Universidad Abierta Interamericana, Rosario, Argentina.

Ramírez Tobón, G. J. (2008). Crianza, cognición y psicopatología en la infancia. Revista Electrónica de Psicología Social Poiésis, 16, 1-8.

Ravera, C. (2009, mayo). Ser niños en los tiempos que ¡corren! Cambios culturales y sus posibles efectos en el desarrollo psicomotor del niño. Trabajo presentado en el 3er. Encuentro Nacional de Psicomotricistas organizado por la Asociación Federal de Psicomotricistas, Córdoba, Argentina.

Raya, A. F., Pino, M. J. \& Herruzo, J. (2009). La agresividad en la infancia: el estilo de crianza parental como factor relacionado. European Journal of Education and Psychology, 2(3), 211222.

Real Academia Española (2001). Diccionario de la lengua española. Madrid: España.

Samaniego, C. V. (2010). Escala de tolerancia parental hacia los comportamientos infantiles, elaboración y validación. Revista Latinoamericana de Psicología, 42(2), 203-214.

Solís-Cámara Reséndiz, P. \& Díaz Romero, M. (2007). Relaciones entre creencias y prácticas de crianza de padres con niños pequeños. Anales de Psicología, 23(2), 177-184.

Spitz, R.A. (1965). El primer año de vida: un estudio psicoanalítico de desarrollo normal y anormal de relaciones de objeto. Nueva York, EE.UU.: Prensa de Universidades Internacional.

Symonds, P.M. (1939). The psychology of parent-child relationships. Nueva York, EE.UU.: AppletonCentury-Crofts.

Triana,A.N., Ávila, L. \& Malagón, A. (2010). Patrones de crianza y cuidado de niños y niñas en Boyacá. Revista Latinoamericana de Ciencias Sociales, Niñez y Juventud, 8(2), 933-945. 